\title{
AVAILABILITY MODELLING OF THE OPTICAL SELF-HEALING RING USING ALGEBRAIC FORMULATION
}

\author{
Katarina RADOŠ \\ H. V. Hrvatinica bb (p. p. 47), 80240 Tomislavgrad, Bosnia and Herzegovina, E-mail: rados.katarina@gmail.com
}

\begin{abstract}
There are several methods for modelling the network availability and one of the most widely used is the enumeration of elementary paths which is based on the principle of mutually exclusive events. In this paper we apply a new method, called - the method of algebraic formulation. This method is based on the operation with algebraic objects (polynomials) and their corresponding transformations. By using this method is developed a model of self-healing WDM ring, which uses wavelength channel protection and calculates the availability of different sized rings. It is also made the calculation of the availability of ring components, links and nodes. Therefore, it is shown how number and length of the links and also the redundancy within the node affect the availability between two nodes within the ring.
\end{abstract}

Keywords: modelling, availability, wavelength channel, self-healing

\section{INTRODUCTION}

Each system consists of elements that are generally not ideal. These elements in the telecommunication networks are the links and nodes that are assumed to break down by accident, independently from each other with the known probabilities and are repairable. Although there are more measures for the availability of the network, in this paper is used availability between two terminals $s$ and $t$ within the network.

Calculation of availability between $s$ and $t$ is reduced to the calculation of the probability that between them there is at least one path that contains the correct links and nodes [1]. Although there are several methods for availability modelling, this paper applies the method of algebraic formulation introduced by prof. Shier [4] that is based on work with polynomials and their transformations instead of working with numerical probabilities. On the example of simple network, he proved to give the same expression for availability between two nodes no matter which method is used. In [5], the author investigates the reliability of the network using exactly the method from reference [4]. Also in [6] the author uses algebraic formulation as one of the methods for calculation the reliability of the network.

This paper presents a method of algebraic formulation and its application in modelling of the availability of selfhealing WDM ring that uses protection of wavelength channel [5].

\section{AVAILABILITY AND UNAVAILABILITY IN GENERAL}

The availability $A$ is often used to describe the performances of system and is defined as the probability that the system is working properly at some point in time $t$ under condition that system was working properly at time $t=0$ and appear incorrectly states (maintenance or failures), which are always fixed and return the system in working state [2]. Also the availability of a system in period of time is defined as the ratio of time for which the system is correct in relation to the total time. If known average time to failure MTTF (Mean Time To Failure) and average time to repair MTTR (Mean Time To Repair), availability can be calculated according to following expression:

$$
A=\frac{M T T F}{M T T F+M T T R}
$$

Usually, MTTF is not known, so the term in the denominator is defined as average time between failures (MTBF-Mean Time Between Failures) [2] and the availability can be written as:

$$
A=\frac{M T T F}{M T B F}
$$

Unavailability $U$ is the probability that is complementary to the availability, i.e. $U=1-A$ [3], respectively that is probability that system does not work properly in some point of time

$U=\frac{M T T R}{M T T F+M T T R} \cong \frac{M T T R}{M T T F} \cong \lambda \cdot M T T R$

where $\lambda$ is the intensity of failure, that is expressed in FIT (Failure In Time, 1FIT $=1$ failure in $10^{9}$ hours).

In reporting on system performance, unavailability is often expressed as the average time of failure MDT (Mean Down Time) [3]

$M D T=3652460 U[\mathrm{~min} /$ year $]$

\section{METHOD OF ALGEBRAIC FORMULATION}

Let some network consists of $V$ nodes and $E$ links connecting these nodes and which are repairable and whose failures happen by accident. $G=(V, E), E=$ $\left\{e_{1}, e_{2}, \ldots, e_{m}\right\}$ and nodes are ideal. $x_{i}$ is the event that indicates that $e_{i}$ is in working state, $A_{i}=p\left[x_{i}\right]$ and events $x_{1}, x_{2}, \ldots, x_{m}$ are independent. Availability between two terminals is an algebraic expression in which the variables $\left\{x_{1}, x_{2}, \ldots, x_{m}\right\}$ use following operations [5]:

1. $x_{i}$ is the event for each $1 \leq i \leq m$ 
2. If $x$ is event, then:

a. $\bar{x}$ is complementary event

b. $1-x$ (complete set minus event)

3. If $x_{1} i x_{2}$ are events, then:
a. $x_{1}+x_{2}$ is union of mutually exclusive events
b. $x_{1} \cdot x_{2}=x_{1} x_{2}$ is the case of two independent events
c. $x_{1} \oplus x_{2}$ is union of arbitrary events
d. $x_{1} \otimes x_{2}$,the product of" arbitrary events

The application of these two operators to serial $(\otimes)$ and parallel $(\oplus)$ structure allows calculation of the availability between two terminals $s$ and $t$, which is represented by a polynomial function of $x$ elements [4]

$A_{s t}(\times)=A_{s t}\left(x_{1}, x_{2}, \ldots, x_{m}\right)$

Which gives the exact availability between the two terminals if the corresponding variables $x_{k}$ are replaced by corresponding availability $A_{k}$ [4].

Generally, algebraic „sum“ of two polynomials $f$ i $g$ is defined as [4]:

$$
f \oplus g=f+g-(f \otimes g)
$$

In order to find the connection between algebraic structures and network availability, should be defined $P_{s t}$ as the set of all elementary $s-t$ paths $P$ in network $G$. Furthermore, the value of path $v(P)$ is defined as ,the product of variables that represent links along the path $P$ [4].

$v(P)=\otimes \prod\left\{x_{k}: k \in P\right\}$

In this case, availability $A_{s t}(\times)$ is ,parallel sum“ of values $v(P)$ over all elementary paths from $s$ to $t[4]$

$$
A_{s t}(\times)=\bigoplus \sum_{P \in P_{s t}} v(P)
$$

\section{AVAILABILITY OF LINKS AND NODES}

Using the previously mentioned mathematical base, availability model is developed and is performed the correct term for the availability between the two terminals for optical unidirectional self - healing ring with protection at the level of wavelength channels (Optical Path Switched Ring Unidirectional or Optical Channel Dedicated Protection Ring OCh-DPRing).

\subsection{Availability of the link}

Link in WDM network consists of: Multiplexer/ Demultiplexer (MD), optical amplifier (BOA-Booster Optical Amplifier), optical cable (OC) and optical preamplifier (OPA) [10] and is shown on Fig. 1:

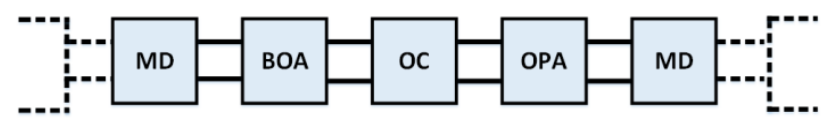

Fig. 1 The scheme of the optical link
This link represents the serial connection of elements so its value is [11]:

$x_{l_{y N P}}=x_{M D} \otimes x_{B O A} \otimes x_{O C} \otimes x_{O P A} \otimes x_{M D}$

As members in the expression (9) have no common elements, instead $\otimes$ operator can be used the sign of "ordinary" multiplication and with $x_{B O A} \cdot x_{O P A}=x_{O A}$ (to simplify), the availability of the link is calculated by simply replacing the variables with corresponding availabilities:

$A_{l_{N P}}=A_{O A} \cdot A_{O C} \cdot A_{M D}^{2}$

\subsection{Availability of the nodes}

According to the most recent works [9], in the nodes within the ring are most commonly used optical add / drop multiplexers and in the interconnect nodes of more rings optical switches. This paper defines two types of nodes in dependence on a signal path., in which add/drop multiplexers are used (Fig. 2).

Final node: $\quad x_{n t}$

Switching node: $x_{n c}$

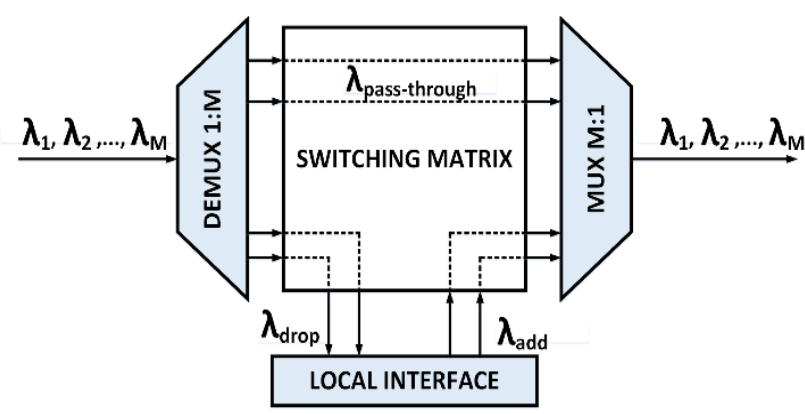

Fig. 2 Simplified architecture of optical add/drop multiplexer

Nodes in which is added or excepted transport signal are called final nodes. In final nodes, the transport signal passes through a local interface $\mathbf{L I}$ and the switching matrix $\mathbf{S W}$ so the value of node is $x_{n t}=x_{L I} \otimes x_{S W}$. The local interface consists of transmitter $\mathbf{T}$ and receiver $\mathbf{R}$ : $x_{L I}=x_{T} \otimes x_{R}$ so $x_{n t}=x_{T} \otimes x_{R} \otimes x_{S W}$.

Nodes in which the transport signal passes from one side to another side are called switching nodes whose value is $x_{n c}=x_{S W}$.

The availability of nodes without redundancy is

$A_{n t}=A_{T} \cdot A_{R} \cdot A_{S W}$

$A_{n c}=A_{S W}$

and in case of redundancy of the switching matrix, the availability is

$A_{n t}=A_{T} \cdot A_{R} \cdot A_{S W} \cdot\left(2-A_{S W}\right)$

$A_{n c}=A_{S W} \cdot\left(2-A_{S W}\right)$ 


\section{MODELLING AVAILABILITY OF WDM RING WITH PROTECTION ON LEVEL OF WAVELENGTH CHANNEL}

In this section is modelled the availability between two nodes of ring considering one or more link failures of working path [7]. Availability between two nodes of the ring is based exclusively on the availability of nodes and links through which is transmitted the number of wavelength channels enabled by the switching matrix. Individual wavelength channels are not especially modelled because in real networks is confirmed that the failure on the optical cable, as connection between two nodes, in $100 \%$ of cases leads to interruption of all fibers in the cable or all wavelength channels that are transmitted over the link [8].

\subsection{OCh-DPRing}

Availability model between two terminals of OChDPRing is based on the availability of working path that the signal passes when there is no failures and the availability of recovery path that signal passes when there is failure (failures) on working path.

\subsubsection{Working path}

The general model of self-healing ring that uses the protection of wave channel is considered (Fig. 3). Transport signal on working path $P_{w}$ passes $m$ links and $m-1$ nodes so the value of working path is:

$$
\begin{aligned}
v_{s t}\left(P_{w}\right)=\otimes \prod_{y, z \in P_{w}} & x_{l y} \cdot x_{n z}= \\
= & {\left[\otimes \prod_{y \in P_{w}} x_{l y}\right]\left[\otimes \prod_{z \in P_{w}} x_{n z}\right] }
\end{aligned}
$$

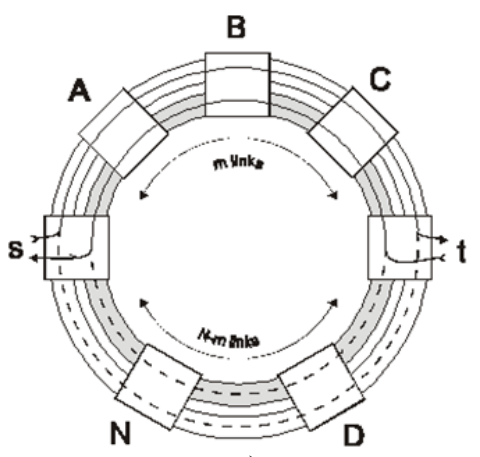

a)

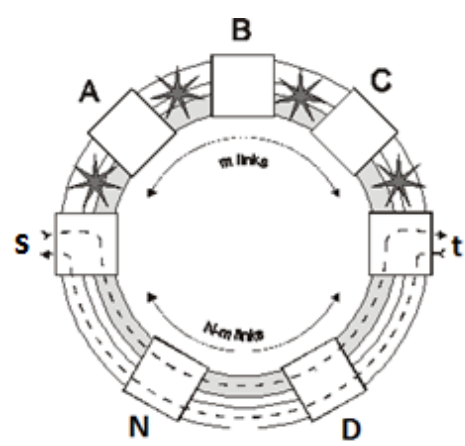

b)

Fig. 3 a) Ring without failures, b) failure on all links of working path
Assuming that all nodes of the same type are equal, the value of working path is:

$$
v_{s t}\left(P_{w}\right)=\left[\left(x_{n t}\right)^{2} \cdot\left(x_{n c}\right)^{m-1}\right]\left[\otimes \prod_{y \in P_{w}}^{m} x_{l y}\right]
$$

\subsubsection{Protection path}

In case of failure (failures) in the network on any optical link of working path, recovery path becomes protection path $P_{P}$ which consists of $N-m$ links and $N-m-1$ transient nodes so the value of the protection path is

$$
\begin{aligned}
v_{s t}\left(P_{P}\right)=\otimes \prod_{y, z \in P_{P}} x_{l y} x_{n z}= \\
\quad\left[\otimes \prod_{y \in P_{P}} x_{l y}\right]\left[\otimes \prod_{z \in P_{P}} x_{n z}\right]
\end{aligned}
$$

The availability of transmission signal between $s$ and $t$ is completely determined with these two paths so the availability between two ring terminals is

$$
\begin{aligned}
& A_{s t, o c h}(\mathrm{x})=\oplus \sum_{P_{i} \in P_{s t}} v_{s t}\left(P_{i}\right)=v_{s t}\left(P_{w}\right) \oplus v_{s t}\left(P_{P}\right)= \\
& v_{s t}\left(P_{w}\right)+v_{s t}\left(P_{P}\right)-v_{s t}\left(P_{w}\right) \otimes v_{s t}\left(P_{P}\right)
\end{aligned}
$$

If, for this link that is used for protection of wavelength channel, instead $x_{l y}$ places its value and assuming that all optical cables have the same length respectively if is $x_{O C_{y}}=x_{O C}, \forall y$ or if average length of cable section in ring is used and if there is no common components among the elements of the link, the expression for the availability becomes:

\section{$A_{s t, o c h}=$}

$\left(A_{n t}\right)^{2}\left[\left(A_{n c}\right)^{m-1}\left(A_{B O A} \cdot A_{O P A}\right)^{m}\left(A_{M D}\right)^{2 m}\left(A_{O C}\right)^{m}+\right.$ $\left(A_{n c}\right)^{N-m-1}\left(A_{B O A} \cdot A_{O P A}\right)^{N-m}\left(A_{M D}\right)^{2(N-m)}\left(A_{O C}\right)^{N-m}-$ $\left.\left(A_{n c}\right)^{N-2}\left(A_{B O A} \cdot A_{O P A}\right)^{N}\left(A_{M D}\right)^{2 N}\left(A_{O C}\right)^{N}\right]$

\section{CALCULATION OF THE AVAILABILITY}

\subsection{OCh-DPRing}

Table 1 shows the input data (intensity of failures and average time to repair) and calculated availability that is later used in calculation of the availability between two ring terminals. On the basis of the expression $W * 25$ [12] is calculated the intensity of the failures for multiplexer/demultiplexer where $W$ is the number of wavelength channels. The intensities of the failures for optical amplifiers are taken from the reference [13].

Table 1 Availability of the optical link components

\begin{tabular}{|c|c|c|c|}
\hline Component & $\lambda(\mathrm{FIT})$ & MTTR $(\mathrm{h})$ & $\mathrm{A}$ \\
\hline$M D\left(40 \lambda_{w c}\right)$ & 1000 & 4 & 0,9999960 \\
\hline$B O A$ & 2000 & 4 & 0,9999920 \\
\hline$P O A$ & 2000 & 4 & 0,9999920 \\
\hline$O C$ & 114 & 10 & 0,9999998 \\
\hline
\end{tabular}

Using these data are calculated availability and average time of failure for links with different lengths (Table 2). 
Table 2 Availability and MDT for links with different lengths

\begin{tabular}{|c|c|c|}
\hline $\begin{array}{c}\text { Link without } \\
\text { protection }\end{array}$ & $\mathrm{A}$ & MDT (min/year) \\
\hline $\mathrm{d}=20 \mathrm{~km}$ & 0,99995320 & 24,59732576 \\
\hline $\mathrm{d}=40 \mathrm{~km}$ & 0,99993040 & 36,57989853 \\
\hline $\mathrm{d}=60 \mathrm{~km}$ & 0,99990760 & 48,56192494 \\
\hline
\end{tabular}

As can be seen in Table 2, the shortest link has the highest availability respectively the lowest unavailability as expected because it represents a serial structure in which the availability of one element just depends on the length of the link.

\subsection{Calculation of the nodes availability}

Data about availability of the node components are taken from the reference [12] in which is given the expression for calculation of failures intensity of the switching matrix as $21 * W * W / 4$.

In Table 3 are shown availability and MDT for the different types of nodes that have no redundancy of switching matrix.

Table 3 Availability and MDT for different types of node $(\mathrm{MTTR}=4 \mathrm{~h})$

\begin{tabular}{|c|c|c|}
\hline Node type & A & MDT min/year) \\
\hline Final & 0,99996537 & 18,19776251 \\
\hline Switching & 0,99996640 & 17,65956663 \\
\hline
\end{tabular}

In practice, often is used redundancy of switching matrix because it is a key element, improvement of the availability of the node in that case is shown in Table 4.

Table 4 Availability and MDT for different types of node with redundancy $(\mathrm{MTTR}=4 \mathrm{~h})$

\begin{tabular}{|c|c|c|}
\hline Node type & A & MDT (min/year) \\
\hline Final & 0,99999897 & 0,53880729 \\
\hline Switching & 0,99999999 & 0,00059334 \\
\hline
\end{tabular}

If we compare the obtained results, it can be seen significant improvement of the availability that is achieved with redundancy $1+1$ of switching matrix. Since the final nodes are common no matter what is the number of the nodes in the ring, failure on key elements of these nodes leads to disconnection and that is the reason why most of the operators use redundancy of switching matrix.

\subsection{Calculation of the ring availability}

Calculation of the availability between two nodes is made under the assumption that links in the network do not have any common elements, respectively there is no common optical cable for working and protection path in the ring. First is calculated the availability between two terminals for one wavelength channel depending on the length of links and with nodes without redundancy of switching matrix. The calculation is made on the rings of 4 and 16 nodes and lengths of the links: 20, 40 and $60 \mathrm{~km}$.

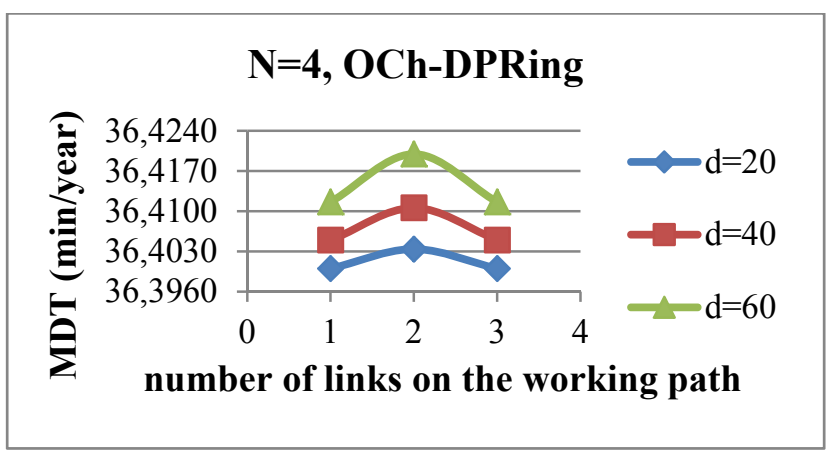

Fig. 4 Unavailability depending on the length of link for the ring of 4 nodes

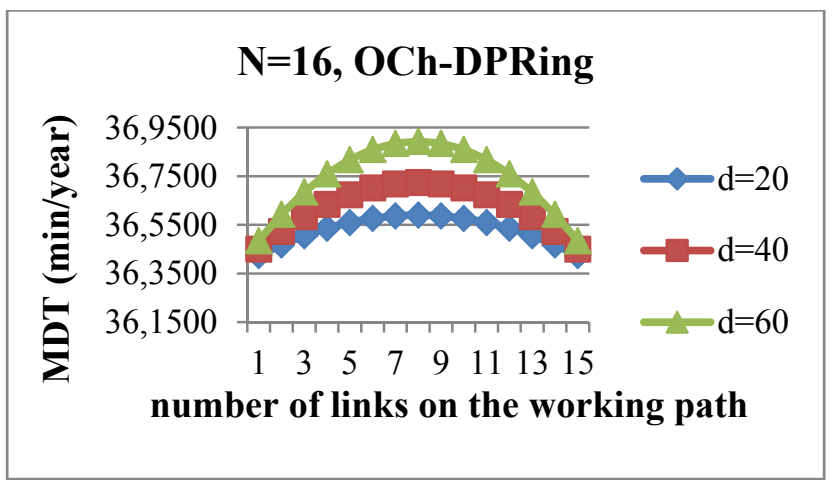

Fig. 5 Unavailability depending on the length of link for the ring of 16 nodes

As seen on Figures 4 and 5, unavailability increases with by increasing the length of links for the same number of nodes. For example, for the ring OCh-DPRing of 4 nodes and link length of $20 \mathrm{~km}$, unavailability is 36,4034 $\mathrm{min} /$ year. For the same type of the ring and link length of $60 \mathrm{~km}$, unavailability is $36,4200 \mathrm{~min} /$ year what gives the difference of $0,0166 \mathrm{~min} /$ year. Unavailabilty for the ring of 16 nodes is higher because of larger total length of the links.

The impact of the unavailability of the final nodes on unavailability between two terminals for OCh-DPRing with the one link of working path for the rings of 4,8 and 16 nodes is shown in Table 5.

For all three sizes of the rings impact of final node's unavailability to the total unavailability between the two terminals is over $99.9 \%$, what was expected because the remaining part of the ring is parallel structure of two branches with nodes and links whose availability is very close 1 .

Table 5 MDT of the final nodes and remaining part of the ring

\begin{tabular}{|c|c|c|c|}
\hline $\mathrm{m}=1$ & $\mathrm{~N}=4$ & $\mathrm{~N}=8$ & $\mathrm{~N}=16$ \\
\hline $\begin{array}{c}\text { MDT of the final } \\
\text { nodes }\end{array}$ & 36,3948 & 36,3948 & 36,3948 \\
\hline $\begin{array}{c}\text { MDT of remaining } \\
\text { part of the ring }\end{array}$ & 0,0051 & 0,0130 & 0,0288 \\
\hline MDT total & 36,4000 & 36,4079 & 36,4237 \\
\hline $\begin{array}{c}\text { Percentage of MDT's } \\
\text { final on the total } \\
\text { MDT }\end{array}$ & 99,9857 & 99,9640 & 99,9206 \\
\hline
\end{tabular}


If redundancy of switching matrix within the nodes (and final and switching) is included, unavailability between two terminals is significantly reduced (see Fig. $6)$.

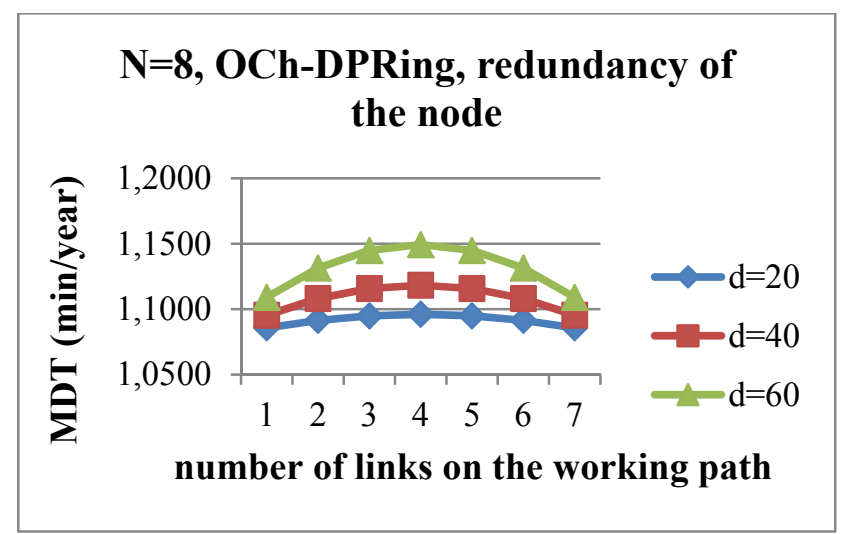

Fig. 6 Unavailability for different lengths of the links with redundancy of the nodes

Unavailability of the ring with redundancy of the switching matrix within nodes, for example link length of $60 \mathrm{~km}$, is decreased by 31,7 times in relation to the unavailability of the ring without redundancy of the switching matrix within nodes.

Calculation results show that the shortest links have the smallest unavailability. Also is shown that the unavailability increases with length of the link, number of links on the working path and with an enlargement of total number of the nodes in the ring. It is also noticeable that the largest unavailability is when the number of links of the working path is half of the total number of the links. Unavailability between two terminals is reduced by applying redundancy of switching matrix within the node. The difference between the largest and the smallest unavailability for all three sizes of the rings are negligible (for ring OCh-DPRing of 8 nodes with redundancy, that difference is $0.0104 \mathrm{~min} /$ year) so it can be said that for these typical sizes of the rings smallest availability is actually equal to the actual availability between two terminals.

\section{CONCLUSION}

Using the method of algebraic formulation in modelling availability of the self-healing WDM ring is shown that the ring, from the availability aspect, is actually parallel structure that consists of two branches which are made of links and nodes. From the analysis of the availability can be conluded that availability of the ring depends on availability of the links and the nodes. Also can be concluded that the availability decreases with an enlargement of: the number of the links on the working path, length of links and number of nodes. The greatest impact on the availability of the ring have the final nodes because in them the signal starts and ends and therefore the failure of the key components of these nodes leads to a breakdown in communication between them. In order to avoid the brekadown in communication, redundancy $1+1$ of the switching matrix as key element is used. That significantly improves the availability between nodes of the ring.

It was also shown that there is a big difference between availability (no matter what is the number of links of the working path) and the minimum availability so that the minimum availability can be used as a parameter in the design of new and in reporting performances of existing networks.

Based on the results obtained in this paper can be said that the ring, from the aspect of the availability, is promising structure for telecom operators as it provides two completely independent paths between nodes and high availability of the connection/services in the network.

\section{REFERENCES}

[1] TORNATORE, M. - MAIER, G. - PATTAVINA, A.: Availability Design of Optical Transport Networks, IEEE Journal on Selected Areas in Communications, Vol. 23, No. 8, August 2005.

[2] CLOUQUEUR, M. A. H.: Availability of Service in Mesh-Restorable Transport Networks, PhD, University of Alberta, 2004.

[3] WANG, X. - PENG, L. - SHEN, G.: Availability Analysis for Elastic Optical Networks with Multipath Virtual Concatenation Technique, Progress In Electromagnetics Research Symposium Proceedings, Guangzhou, China, Aug. 25-28, 2014.

[4] SHIER, D. R.: Network Reliability and Algebraic Structures, Oxford University Press, New York, 1991.

[5] HARMS, D. D.: A symbolic algebra environment for research in network reliability, $\mathrm{PhD}$, Simon Fraser University, September 1992.

[6] JONCZY, J.: Generic Frameworks for the Analysis of Dependable Systems: Algebraic Path Problems, Reliability, and Diagnostics, $\mathrm{PhD}$, University of Bern, 2009, Switzerland.

[7] MELlO, D. A. A. - SCHUPKE, D. A. SCHEFFEL, M. - WALDMAN, H.: Availability maps for connections in WDM optical networks, In International Workshop on the Design of Reliable Communications Networks (DRCN), 2005.

[8] RADOS, I. - TURALIJA, P. - SUNARIC, T.: Suggestions for availability improvement of optical cables, 1st IEEE International Conference on Circuits and Systems for Communications, 2002, Proceedings, ICCSC '02.

[9] SONG, L. - ZHANG, J. - MUKHERJEE, B.: Dynamic Provisioning with Availability for Differentiated Service in Survivasble Mesh Networks, IEEE Journal on Selected areas in communications, Vol. 25, No. 4, April 2007. 
[10] MOHAN, M. - MOHAN, N.: Link Failure Recovery in WDM Networks, International Journal of Computer Science and Electronics Engineering (IJCSEE), Vol. 1, Issue 5, 2013.

[11] PANDA, C. - PATRO, S. N. - GANTAYAT, P. K.: Link Reliability in WDM Optical Network, Indian Journal of Computer Science and Engineering (IJCSE), Vol. 3, No. 1, Feb.-Mar. 2012.

[12] EUN, J. - PARK, G. - AHN, G. - KIM, H.: The Optical Network Design using Availability Models to Accept Additional Nodes, Advanced Engineering Forum Vols. 2-3, 2012, pp. 553-558.

[13] VERBRUGGE, S. - COLLE, D. - DEMEESTER, P. - HUELSERMANN, R. - JAEGER, M.: General
Availability Model for Multilayer Transport Networks, IEEE, 2005.

Received July 27, 2016, accepted October 20, 2016

\section{BIOGRAPHY}

Katarina Radoš was born on 02.09.1992. In July 2016 she graduated (MSc) at the department of Telecommunications and Informatics of the Faculty of Electrical Engineering, Mechanical Engineering and Naval Architecture at University in Split, Croatia. Her scientific research is focusing on optical communications, optical access networks, reliability and availability of optical networks. 